\title{
Biscoitos com diferentes concentrações de farinha de quinoa em substituição parcial à farinha de trigo
}

\author{
Cookies with different concentrations of quinoa flour in partial \\ replacement of wheat flour
}

\section{Autores | Authors}

Marília Alessandra BICK

Centro Universitário Franciscano Área de Ciências da Saúde, Curso de Nutrição CEP 97010-032 Santa Maria/RS - Brasil e-mail:mariliabick@gmail.com

\section{Aline de Oliveira FOGAÇA}

Centro Universitário Franciscano Área de Ciências da Saúde, Curso de Farmácia CEP 97010-032

Santa Maria/RS - Brasil e-mail: alinefogaca@gmail.com

\section{\Cátia Regina STORCK}

Centro Universitário Franciscano Área de Ciências da Saúde, Curso de Nutrição

Rua Silva Jardim, 1175, Centro CEP 97010-032

Santa Maria/RS - Brasi e-mail: catia.sm@gmail.com

ه Autor Correspondente / Corresponding Author

Recebido / Received: 27/09/2013 Aprovado / Approved: 02/06/2014

Publicado / Published: jun./2014

\section{Resumo}

A proposta deste estudo foi desenvolver e avaliar as características nutricionais, físicas e sensoriais de um biscoito, elaborado com substituição parcial da farinha de trigo por farinha de quinoa, além de analisar as características nutricionais das farinhas de trigo e quinoa. Foram elaboradas formulações de biscoito padrão (100\% de farinha de trigo) e com substituição de farinha de trigo nas concentrações de 10\%, 20\% e 30\% de farinha de quinoa. Os biscoitos foram avaliados quanto aos parâmetros químicos, físicos e de textura, além de atributos sensoriais. As farinhas foram avaliadas quanto à composição centesimal. Os atributos de diâmetro, espessura, fator de expansão, volume específico, rendimento e dureza mostraram-se bastante similares entre o biscoito padrão e as formulações com farinha de quinoa, o que indicou que a farinha de quinoa é bem incorporada à massa dos biscoitos. Conforme se aumentou a concentração, de 10 até $30 \%$ de farinha de quinoa, os biscoitos apresentaram maior maciez. Note-se que a nota no teste de aceitabilidade para todos os atributos variou entre seis e sete, não diferindo entre as formulações, indicando que a adição de até $30 \%$ de farinha de quinoa não altera as características sensoriais do biscoito. Assim, a substituição parcial de farinha de trigo por farinha de quinoa incrementou o valor nutricional dos biscoitos. A composição centesimal da farinha mostrou que a quinoa apresenta maiores teores de proteína, lipídios, fibras e cinzas, em comparação ao trigo.

Palavras-chave: Biscoitos; Farinha de quinoa; Valor nutricional; Atributos sensoriais.

\section{Summary}

The purpose of this study was to develop and evaluate the nutritional, physical and sensory characteristics of a cookie made with partial replacement of the wheat flour by quinoa flour, and to analyze the nutritional characteristics of the wheat and quinoa flours. Formulations were drawn up of a biscuit with $100 \%$ of wheat flour and with replacement of the wheat flour by quinoa flour at concentrations of $10 \%, 20 \%$ and $30 \%$. The cookies were evaluated with respect to their chemical, physical and texture parameters and sensory attributes, and the flours for their proximate composition. The attributes of diameter, thickness, spreading factor, specific volume, hardness and yield were quite similar between the standard cookie and the formulations with quinoa flour, indicating that the quinoa flour was well incorporated into the biscuit dough. As the concentration increased from 10 to $30 \%$ of quinoa flour, the hardness of the cookies decreased, and the score in the acceptability test for all the attributes ranged between six and seven. There was no difference between the formulations showing that the addition of up to $30 \%$ quinoa flour did not change the sensory characteristics of the cookies. Thus, the partial replacement of wheat flour by quinoa flour increased the nutritional value of the cookies. The chemical composition of the flours showed that the quinoa flour presented higher protein, fat, fibre and ash contents than the wheat flour.

Key words: Cookies; Quinoa flour; Nutritional value; Sensory attributes. 


\section{Introdução}

A quinoa (Chenopodium quinoa) é considerada um pseudocereal, por não fazer parte da família das gramíneas, mas produz grãos que podem ser utilizados como cereais ou moídos, para utilização em forma de farinha (BRADY et al., 2007). Sua principal característica é seu elevado teor de proteínas e aminoácidos essenciais, que não são comuns em outros cereais, como a lisina (44 mg aa/g proteína), o triptofano (12 mg aa/ g proteína), a histidina (36 mg aa/ g proteína) e a valina (76 mg aa/ g proteína) (STIKIC et al., 2012). O grão de quinoa contém aproximadamente $77 \%$ de carboidratos, $12 \%$ de proteínas, 6,5\% de lipídios e 3\% de minerais (HIROSE et al., 2010); a quinoa é considerada, segundo a Organização Mundial de Saúde (OMS), como um alimento completo, por sua composição nutricional ser superior à dos demais cereais utilizados na alimentação humana (SILVA et al., 2011).

Estudos demonstram que a quinoa é uma boa fonte de fibras alimentares e pode substituir os cereais comuns (REPO-CARRASCO-VALENCIA e SERNA, 2011). Os carboidratos presentes neste pseudocereal são considerados funcionais, pois têm a propriedade de melhorar o controle glicêmico, os triglicérides e os níveis de lipídeos plasmáticos (FARINAZZI-MACHADO et al., 2012). Assim, o mesmo pode ser considerado um alimento funcional.

Alimentos funcionais são benéficos à saúde, pois reduzem o risco de diversas doenças, entre as quais as cardiovasculares, o câncer e as doenças neurodegenerativas. Tal propriedade está relacionada com a atividade antioxidante, que é atribuída, em grande parte, aos compostos fenólicos (HIROSE et al., 2010). Os polifenóis estão amplamente presentes em alimentos de origem vegetal, divididos principalmente entre flavonoides, ácidos fenólicos e taninos, que são antioxidantes. Os níveis de flavonoides presentes na quinoa (58 mg/100 g) (REPO-CARRASCO-VALENCIA et al., 2010) são superiores ao do mirtilo (47 mg. $100 \mathrm{~g}^{-1}$ ) (SOUZA et al., 2014), uma fruta sabidamente rica neste composto.

A quinoa pode ser encontrada em forma de farinha, flocos e grãos, produtos estes que são utilizados na produção de diversos produtos alimentícios (FARINAZZI-MACHADO et al., 2012). Embora não seja largamente utilizada na alimentação da população em geral, é bastante utilizada para celíacos e vegetarianos, devido à ausência de glúten e ao alto valor biológico das proteínas, quando comparada aos outros cereais (CALDERELLI et al., 2010).

Atualmente, os consumidores estão mais conscientes de suas escolhas alimentares e, por isso, buscam produtos com valor agregado e, em geral, alimentos mais nutritivos (BRADY et al., 2007). Para isso, é necessário o desenvolvimento de novos produtos que sigam estas tendências (VILLARROEL et al., 2009). Devido ao tempo de vida de prateleira e à aceitabilidade pela população, os biscoitos, mesmo não sendo produtos básicos utilizados na alimentação, são amplamente aceitos pela população em geral e podem ter as características nutricionais e sensoriais melhoradas através da incorporação de alimentos funcionais (HIROSE et al., 2010).

Baseando-se em evidências de que a quinoa é um alimento rico em nutrientes e com características de alimento funcional, torna-se interessante desenvolver um produto prático e com características sensoriais aceitáveis. Assim, objetivou-se desenvolver um biscoito com incorporação de farinha de quinoa na farinha de trigo.

\section{Material e métodos}

\subsection{Amostras}

Os ingredientes - farinha de trigo, açúcar refinado, ovo, óleo vegetal, fermento químico e sal refinado - para elaboração do biscoito foram adquiridos no comércio local do município de Santa Maria-RS, sendo a farinha de quinoa produzida na Bolívia. Os ingredientes não perecíveis foram armazenados em temperatura ambiente $\left(24^{\circ} \mathrm{C}\right)$, longe de contaminantes e da exposição à luz, e os ingredientes perecíveis, armazenados em temperatura refrigerada $\left(4^{\circ} \mathrm{C}\right)$

\subsection{Formulação dos biscoitos}

A elaboração dos biscoitos foi realizada de acordo com a Tabela 1, em triplicata. Foi elaborada uma formulação padrão (100\% farinha de trigo) e outras com substituição parcial da farinha de trigo por 10\%, 20\% e $30 \%$ de farinha de quinoa.

Tabela 1. Formulação dos biscoitos sem e com adição de farinha de quinoa.

\begin{tabular}{lcccc}
\multicolumn{1}{c}{ Ingredientes } & Padrão & $\mathbf{1 0 \%}$ & $\mathbf{2 0} \%$ & $\mathbf{3 0 \%}$ \\
Farinha de Trigo (g) & 100 & 90 & 80 & 70 \\
Farinha de Quinoa (g) & 0 & 10 & 20 & 30 \\
Açúcar Refinado (g) & 40 & 40 & 40 & 40 \\
Ovos (g) & 40 & 40 & 40 & 40 \\
Óleo Vegetal (mL) & 12 & 12 & 12 & 12 \\
Fermento Químico (g) & 0,8 & 0,8 & 0,8 & 0,8 \\
Sal Refinado (g) & 0,7 & 0,7 & 0,7 & 0,7 \\
\hline
\end{tabular}


Para a elaboração da massa, misturaram-se manualmente os ingredientes secos e, a seguir, foram adicionados o ovo e o óleo. A massa foi homogeneizada por cinco minutos e armazenada em refrigeração $\left(4^{\circ} \mathrm{C}\right)$ durante uma hora. Após abertura da massa, os discos (50 $\mathrm{mm}$ de diâmetro e $7 \mathrm{~mm}$ de espessura) foram submetidos ao forneamento a $180^{\circ} \mathrm{C}$, durante 15 minutos, em forno a gás (Marca Venâncio - Ciclone digital), sendo antes pesados e medidos os diâmetros e as espessuras. Após resfriamento, os biscoitos foram avaliados quanto às propriedades físicas e de textura. Para análises de composição centesimal, fenóis totais e atividade antioxidante, os biscoitos utilizados foram levados à estufa (De Leo - HW500) a $55^{\circ} \mathrm{C}$, para secagem, e foram moídos (moinho tipo Willye TE-650, marca Tecnal), peneirados e armazenados em potes plásticos vedados em temperatura adequada até o momento das análises. Todas as análises foram realizadas em triplicata.

\subsection{Propriedades físicas dos biscoitos}

As análises foram realizadas de acordo com os procedimentos descritos no método 10-50D da AACC (2000), para a determinação de diâmetro, espessura, fator de expansão e volume específico. O rendimento foi calculado a partir do peso cru e do peso assado, em percentual. Estas análises foram realizadas em amostras aleatórias, constituídas de nove biscoitos provenientes de três fornadas, para cada tratamento.

A dureza dos biscoitos assados foi avaliada em texturômetro TA.XT.plus texture analyser, utilizando o software Exponent Stable Micro Systems. Para o teste, foi utilizado o probe 3-Point bend Rig (HDP/3PB), para partir o biscoito ao meio. As condições de teste foram: velocidade de pré-teste $1 \mathrm{~mm} \cdot \mathrm{s}^{-1}$, pós-teste $10 \mathrm{~mm} \cdot \mathrm{s}^{-1} \mathrm{e}$ de teste $3 \mathrm{~mm} \cdot \mathrm{s}^{-1}$, e força de contato de $50 \mathrm{~g}$. A análise foi realizada com sete amostras de biscoitos de cada formulação, após 24 horas do forneamento.

\subsection{Análise da composição centesimal}

Determinou-se a composição centesimal da farinha de trigo, da farinha de quinoa e dos biscoitos prontos. O conteúdo de umidade (estufa a $105^{\circ} \mathrm{C}$ por 24 horas), o teor de nitrogênio total (fator 6,25) (método n 46-13) e o teor de cinzas (método $n^{\circ}$ 08-01) foram determinados de acordo com a AACC (1995). O teor de lipídios foi determinado de acordo com a AOAC (CUNNIFF, 1995) (éter de petróleo como solvente). A quantificação de fibra bruta foi determinada pelo método da fibra bruta (BRASIL, 1991). Os carboidratos foram calculados pela diferença dos demais componentes. Os resultados foram expressos em base úmida. O valor energético foi calculado utilizando-se os seguintes fatores de conversão de Atwater: carboidratos 4 kcal.g-1 ${ }^{-1}$ proteínas 4 kcal.g-1 e lipídios 9 kcal.g-1 (MENDEZ et al., 1995).

\subsection{Análise de fenóis totais e atividade antioxidante}

Para obtenção dos extratos, foram realizadas duas extrações, sendo a primeira com uma solução de metanol $80 \%$ e a segunda com uma solução de acetona $80 \%$. Este extrato bruto foi utilizado nas dosagens de fenóis totais e de atividade antioxidante. Os compostos fenólicos foram determinados por espectrofotômetro, marca PRÓ-ANÁLISE ${ }^{\circledR}$ e modelo UV-1100. A concentração de polifenóis totais foi determinada segundo o método colorimétrico Folin-Ciolcateau, descrito por Singleton e Rossi Junior (1965). Para quantificação, foi empregada uma curva padrão de ácido gálico e o teor de polifenóis totais foi expresso em equivalentes de ácido gálico (mg. $\left.100 \mathrm{~g}^{-1}\right)$.

A atividade antioxidante foi determinada utilizando-se o método DPPH, de acordo com Rufino et al. (2007), e os resultados expressos em porcentagem de sequestro de radicais livres (\%SRL). A quantidade de amostra utilizada foi de $2 \mathrm{~g}$, a qual foi diluída para $25 \mathrm{~mL}$. Os compostos fenólicos e a atividade antioxidante foram determinados na farinha de quinoa e nos biscoitos.

\subsection{Análise sensorial}

A análise sensorial foi realizada apenas com os biscoitos adicionados de farinha de quinoa, por meio do teste de aceitação, utilizando-se escala hedônica estruturada de nove pontos, variando entre os extremos: (9) - gostei extremamente e (1) - desgostei extremamente (DUTCOSKY, 2011). Para avaliar a intenção de compra, utilizou-se uma escala variando de (5) certamente compraria a (1) certamente não compraria (ZENEBON e PACUET, 2008). Os atributos avaliados foram: aparência, cor, aroma, textura e sabor.

A análise sensorial foi realizada no Laboratório de Técnica Dietética do Centro Universitário Franciscano. Participaram da análise 50 provadores não treinados, de ambos os sexos, que assinaram o Termo de Consentimento Livre e Esclarecido, conforme Resolução 196/96 do Conselho Nacional de Saúde. As amostras foram servidas em blocos completos casualisados, codificadas com números aleatórios de três dígitos. O trabalho foi aprovado pelo Comitê de Ética e Pesquisa do Centro Universitário Franciscano, sob $n^{\circ} 211.809$.

\subsection{Análise estatística}

Os resultados foram avaliados através de análise de variância (ANOVA) e as médias foram comparadas pelo teste de Duncan no nível de 5\% de significância, utilizando-se o software SPSS (Statistical Package for the Social Sciences) 14.0. 


\section{Resultados e discussão}

\subsection{Propriedades físicas dos biscoitos}

A Tabela 2 apresenta as médias dos dados obtidos nas avaliações das variáveis 'diâmetro cru' (DC) e 'diâmetro após forneamento' (DA), 'fator de expansão' (FE), 'rendimento' (R), 'volume específico' (VE) e 'dureza' (D) dos biscoitos.

Não é esperado que o diâmetro após o forneamento seja maior do que o original, pois isto resultaria em perda do formato de corte. Todos os biscoitos apresentaram aumento no diâmetro após o forneamento; no entanto, a substituição pela farinha de quinoa parece não ter influenciado esse parâmetro. A avaliação do diâmetro cru mostra que o tratamento padrão não obteve diferença significativa entre as formulações, demonstrando, desta forma, um elevado grau de similaridade física entre os biscoitos padrão e aqueles adicionados de quinoa. Apenas a formulação com 30\% de farinha de quinoa diferiu dos demais quanto ao diâmetro cru, devido à diminuição do teor de glúten, deixando a massa com menor firmeza, não mantendo o diâmetro de corte. Quanto ao diâmetro assado, as formulações padrão e com $20 \%$ de incorporação diferiram significativamente no nível de $5 \%$ entre si; porém, os mesmos não sofreram influência significativa em relação aos tratamentos 10 e 30\% de farinha de quinoa.

Moraes et al. (2010) relatam que, ao formularem biscoitos com farinha de trigo mole, observaram o aumento no diâmetro dos biscoitos após o forneamento, o que foi atribuído ao baixo conteúdo de glúten e à força da massa, a qual forma um filme frágil em vez de uma rede viscoelástica. Pode-se considerar que a adição de farinha de quinoa reduz a rede viscoelástica criada pelo glúten, em função da ausência desta proteína no pseudocereal.

Ocorreu variação significativa $(p<0,05)$ entre 0 fator de expansão encontrado nos biscoitos produzidos com $10 \%$ e $30 \%$ de quinoa, sendo que, em relação ao biscoito padrão e com $20 \%$ de quinoa, não foi observada alteração significativa. Biscoitos com fator de expansão muito alto ou muito baixo causam problemas na indústria, resultando em produtos com tamanho pequeno ou peso muito elevado (FERREIRA et al., 2009). O volume específico e o rendimento também não foram alterados em função da adição da farinha de quinoa. Note-se que apenas houve diferença no volume específico entre os biscoitos com $10 \%$ e $20 \%$ de farinha de quinoa.

Fasolin et al. (2007) desenvolveram biscoitos com adição de farinha de banana e observaram que as formulações apresentaram maior diâmetro após forneamento, o que indica que a adição de farinha de banana pode ter interferido na textura da massa, fazendo com que a mesma sofresse maior espalhamento durante a cocção e, consequentemente, aumentado o diâmetro. Assis et al. (2009) também encontraram maior diâmetro após forneamento, quando desenvolveram um biscoito com farinha de aveia, o que atribuíram ao baixo conteúdo de glúten e à força da farinha de trigo.

A dureza dos biscoitos, avaliada por método instrumental, é proporcional à força aplicada para ocasionar uma deformação. A força máxima avaliada é dependente da formulação (qualidade da farinha, quantidade de açúcares e gorduras), da umidade do biscoito e da conservação. A dureza é um dos fatores que determinam a aceitabilidade dos alimentos pelo consumidor e, assim, é desejável que seus valores não sejam muito altos (ASSIS et al., 2009).

Os valores da análise da textura instrumental estão apresentados na Tabela 2, na qual se observou que, conforme se aumentou a concentração de farinha de quinoa, houve diminuição significativa da dureza dos biscoitos, provavelmente devido ao enfraquecimento da rede de glúten; nota-se que os tratamentos diferiram significativamente entre si, no nível de 5\%. Moraes et al. (2010) encontraram, em seu estudo, que concentrações de gordura entre 30 e $38 \%$ reduzem a dureza de biscoitos e que a redução de até $31 \%$ de açúcar não afeta este parâmetro físico.

\subsection{Composição centesimal, polifenóis totais e atividade antioxidante}

\subsubsection{Farinha de trigo e farinha de quinoa}

A composição química da farinha de trigo e da farinha de quinoa, utilizadas na produção dos biscoitos, está apresentada na Tabela 3. Ao comparar com a

Tabela 2. Propriedades físicas dos biscoitos sem e com adição de farinha de quinoa.

\begin{tabular}{ccccc} 
Tratamento & Padrão & $\mathbf{1 0 \%}$ & $\mathbf{2 0} \%$ & $\mathbf{3 0} \%$ \\
DC $(\mathrm{mm})$ & $50,9 \pm 1,3^{\mathrm{b}}$ & $51,7 \pm 1,0^{\mathrm{ab}}$ & $51,6 \pm 0,8^{\mathrm{ab}}$ & $52,0 \pm 0,4^{\mathrm{c}}$ \\
DA $(\mathrm{mm})$ & $54,6 \pm 1,5^{\mathrm{b}}$ & $55,9 \pm 1,2^{\mathrm{ab}}$ & $56,5 \pm 1,3^{\mathrm{a}}$ & $55,8 \pm 1,2^{\mathrm{ab}}$ \\
FE & $4,51 \pm 0,50^{\mathrm{ab}}$ & $4,18 \pm 0,30^{\mathrm{b}}$ & $4,46 \pm 0,32^{\mathrm{ab}}$ & $4,82 \pm 0,29^{\mathrm{a}}$ \\
R $(\%)$ & $87 \pm 2^{\mathrm{a}}$ & $90 \pm 1^{\mathrm{a}}$ & $87 \pm 1^{\mathrm{a}}$ & $88 \pm 7^{\mathrm{a}}$ \\
VE $\left(\mathrm{cm}^{3} \cdot \mathrm{g}^{-1}\right)$ & $1,72 \pm 0,09^{\mathrm{ab}}$ & $1,70 \pm 0,06^{\mathrm{b}}$ & $1,78 \pm 0,04^{\mathrm{a}}$ & $1,72 \pm 0,06^{\mathrm{ab}}$ \\
D (kgf) & $14,6 \pm 1,03^{\mathrm{a}}$ & $12,4 \pm 0,99^{\mathrm{b}}$ & $10,9 \pm 0,85^{\mathrm{c}}$ & $8,5 \pm 0,86^{\mathrm{d}}$ \\
\hline
\end{tabular}

DC: Diâmetro Cru; DA: Diâmetro Assado; FE: Fator de Expansão; R: Rendimento; VE: Volume Específico; D: Dureza. Média \pm desvio padrão. Letras iguais em uma mesma linha não apresentam diferenças significativas pelo teste de Duncan $(p<0,05)$. 
informação nutricional fornecida pela Tabela Brasileira de Composição Química dos Alimentos (UNICAMP, 2011) para a farinha de trigo, verificou-se semelhança entre os valores encontrados, em nosso estudo, para os teores de valor energético, com $360 \mathrm{kcal}^{1} 100 \mathrm{~g}^{-1}$ na TACO e de 355,2 kcal. $100 \mathrm{~g}^{-1}$ nesta pesquisa. Da mesma forma, para a umidade, com valores de 13 g. $100 \mathrm{~g}^{-1} \mathrm{e}$

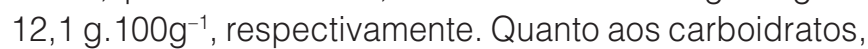
o valor encontrado neste estudo e o valor de referência da tabela foi o mesmo (75 g. $\left.100 \mathrm{~g}^{-1}\right)$. Os valores que apresentaram as maiores diferenças foram da proteína, com 9,8 g. $100 \mathrm{~g}^{-1}$ e $11,1 \mathrm{~g} .100 \mathrm{~g}^{-1}$, e para lipídios, de $1,4 \mathrm{~g} \cdot 100 \mathrm{~g}^{-1}$ e $1,18 \mathrm{~g} \cdot 100 \mathrm{~g}^{-1}$, respectivamente.

Ao compararmos a composição da farinha de quinoa encontrada no estudo de Lopes (2009), observa-se proximidade com os valores encontrados neste estudo, sendo, respectivamente, o valor energético: 352 kcal. $100 \mathrm{~g}^{-1}$ e $349 \mathrm{kcal}^{1} 100 \mathrm{~g}^{-1}$; os carboidratos: $65,0 \mathrm{~g} \cdot 100 \mathrm{~g}^{-1}$ e $65,8 \mathrm{~g} \cdot 100 \mathrm{~g}^{-1}$; a fibra bruta: $3,72 \mathrm{~g} \cdot 100 \mathrm{~g}^{-1}$ e $3,92 \mathrm{~g} \cdot 100 \mathrm{~g}^{-1}$. Os demais quesitos mostraram pequenas diferenças, sendo maiores para proteínas: $11,52 \mathrm{~g} 100 \mathrm{~g}^{-1}$

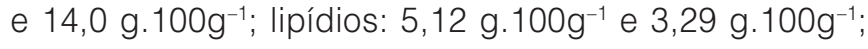

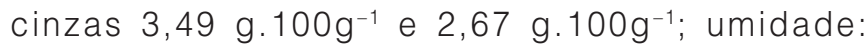
$11,15 \mathrm{~g} .100 \mathrm{~g}^{-1}$ e $10,28 \mathrm{~g} \cdot 100 \mathrm{~g}^{-1}$, respectivamente. Estas diferenças podem ser atribuídas aos métodos utilizados nas análises, bem como à cultivar da planta, já que a quinoa apresenta inúmeras variedades, que diferem entre si quanto aos teores de nutrientes (STIKIC et al., 2012).

Os valores energéticos das farinhas de trigo e quinoa estudados foram muito semelhantes (355,2 e $349 \mathrm{Kcal}$, respectivamente), indicando que a substituição de uma farinha pela outra não ocasiona prejuízos, como a diminuição do valor calórico do alimento.

Conforme os dados obtidos neste estudo, a quantidade de carboidratos na farinha de trigo $\left(75,2 \mathrm{~g} \cdot 100 \mathrm{~g}^{-1}\right)$ é maior do que na farinha de quinoa $\left(65,8 \mathrm{~g} \cdot 100 \mathrm{~g}^{-1}\right)$. James (2009) relata semelhança entre o conteúdo de carboidratos da quinoa (66\%) ao da cevada (68\%) e do arroz (72\%). Os carboidratos da quinoa, constituídos principalmente por amido $(21 \%$ amilose e $79 \%$ amilopectina), fibras solúveis (1,3-6,1\%) e açúcares simples (3\%), como maltose, D-galactose, D-ribose, frutose e glicose, podem ser considerados como alimentos funcionais, em função do baixo índice glicêmico (70\%), pois apresentam efeitos benéficos quanto à hipoglicemia e à indução da redução dos triglicerídeos (JAMES, 2009), quando comparados ao pão branco (100\%).

A farinha de quinoa apresentou um teor de proteína maior do que a farinha de trigo (14 e 11,1 g. $100 \mathrm{~g}^{-1}$, respectivamente). Na literatura, encontram-se valores de 12 a 23\% para a quinoa (JAMES, 2009) e, quando comparada à farinha de outros cereais, a quinoa apresenta maior quantidade de proteína do que a cevada (11\%), o arroz $(7,5 \%)$ e o milho (13,4\%) (CAPRILES et al., 2006).

O teor de lipídios encontrado em nosso estudo foi superior na farinha de quinoa (3,29 g. $\left.100 \mathrm{~g}^{-1}\right)$ (Tabela 3), quando comparada à farinha de trigo $\left(1,18 \mathrm{~g} .100 \mathrm{~g}^{-1}\right)$. Stikic et al. (2012) encontraram 5,8\% de lipídios, estes ricos em ácidos graxos essenciais (linolêico e linolênico).

O teor de cinzas encontrado na farinha de quinoa foi seis vezes maior (2,67 g. $\left.100 \mathrm{~g}^{-1}\right)$ (Tabela 3$)$ do que na farinha de trigo $\left(0,45 \mathrm{~g} \cdot 100 \mathrm{~g}^{-1}\right)$ (Tabela 3 ), sendo que este foi superior ao resultado encontrado por Borges et al. (2003) $\left(2,39 \mathrm{~g} \cdot 100 \mathrm{~g}^{-1}\right)$. A quinoa também apresenta maior conteúdo de cinzas do que o arroz $\left(0,5 \mathrm{~g} \cdot 100 \mathrm{~g}^{-1}\right)$ e demais cereais (milho, trigo, aveia), comumente utilizados na alimentação humana (LOPES, 2009). Este fator, associado ao alto valor biológico da proteína e demais compostos bioativos da quinoa, favorece a aplicação da mesma na fortificação de farinhas e elaboração de produtos, como biscoitos, bolos e pães.

A quantidade de fibra bruta na farinha de quinoa foi 15 vezes superior $\left(3,92 \mathrm{~g} .100 \mathrm{~g}^{-1}\right)$ (Tabela 3 ) à farinha

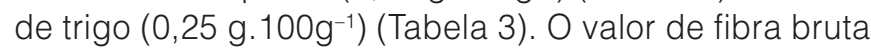
encontrado em pesquisa de Ascheri et al. (2002) foi inferior $\left(3,4 \mathrm{~g} \cdot 100 \mathrm{~g}^{-1}\right)$ ao encontrado em nosso experimento, para a farinha de quinoa. Desta forma, pode-se afirmar que, ao adicionar farinha de quinoa aos alimentos, estaremos

Tabela 3. Composição centesimal, polifenóis totais e atividade antioxidante das farinhas de trigo e de quinoa.

\begin{tabular}{lcc} 
& Farinha de trigo & Farinha de quinoa \\
Valor energético $(\mathrm{kcal} / 100 \mathrm{~g})$ & 355,2 & 349,0 \\
Carboidrato $\left(\mathrm{g} \cdot 100 \mathrm{~g}^{-1}\right)$ & 75,2 & 65,8 \\
Proteína $\left(\mathrm{g} \cdot 100 \mathrm{~g}^{-1}\right)$ & $11,1 \pm 0,71$ & $14 \pm 0,1$ \\
Lipídios $\left(\mathrm{g} .100 \mathrm{~g}^{-1}\right)$ & $1,18 \pm 0,11$ & $3,29 \pm 0,02$ \\
Fibra bruta $\left(\mathrm{g}^{1} 100 \mathrm{~g}^{-1}\right)$ & $0,25 \pm 0$ & $3,92 \pm 1,69$ \\
Cinzas $\left(\mathrm{g} \cdot 100 \mathrm{~g}^{-1}\right)$ & $0,45 \pm 0,09$ & $2,67 \pm 0,04$ \\
Umidade $\left(\mathrm{g} \cdot 100 \mathrm{~g}^{-1}\right)$ & $12,1 \pm 0,13$ & $10,28 \pm 0,24$ \\
Polifenóis totais $\left(\mathrm{g} \cdot 100 \mathrm{~g}^{-1}\right)^{*}$ & - & $139,6 \pm 10,5$ \\
Atividade antioxidante $(\% \mathrm{SRL})$ & - & $27,6 \pm 1,5$ \\
\hline
\end{tabular}

*Em ácido gálico.\%SRL = sequestro de radicais livres na solução a $8 \%$. 
Biscoitos com diferentes concentrações de farinha de quinoa em substituição parcial à farinha de trigo

BICK, M. A. et al.

aumentando a quantidade de fibra disponível, o que pode contribuir para a redução da concentração de lipídios e glicose no sangue, aumentando a sensibilidade à insulina e diminuindo a pressão sanguínea, bem como colaborando para a redução de peso. Segundo a OMS (2003), para este nutriente, recomenda-se o consumo diário de 20 a 30 gramas; dessa forma, o consumo de 100 gramas de farinha de quinoa supre em 16\% as necessidades diárias de fibra de um indivíduo saudável.

A umidade é requisito básico para garantia da qualidade de farinhas, que deve apresentar um limite máximo de 15\%, segundo a RDC 263/2005 (BRASIL, 2005). Desta forma, pode-se dizer que o valor de umidade encontrado nas farinhas utilizadas está dentro dos padrões recomendados.

O resultado encontrado quanto ao teor de compostos fenólicos da farinha de quinoa (Tabela 3) foi superior aos encontrados na polpa de maracujá $\left(20,0 \mathrm{mg} .100 \mathrm{~g}^{-1}\right)$, goiaba $\left(83,0 \mathrm{mg} .100 \mathrm{~g}^{-1}\right)$, abacaxi $\left(21,7 \mathrm{mg} 100 \mathrm{~g}^{-1}\right)$, mas foi menor do que os valores encontrados para a polpa de manga $\left(544,9 \mathrm{mg} \cdot 100 \mathrm{~g}^{-1}\right)$ e acerola (580,1 mg. $100 \mathrm{~g}^{-1}$ ) (KUSKOSKI et al., 2006). Costa et al. (2012) apresentaram, em seu estudo, valores de $108,91 \mathrm{mg} .100 \mathrm{~g}^{-1}$ para farinha de jatobá e de $167,66 \mathrm{mg} \cdot 100 \mathrm{~g}^{-1}$ para farinha de pequi. Quando comparados ao valor encontrado em nosso estudo, de 139,6 mg. $100 \mathrm{~g}^{-1}$ (Tabela 3), nota-se que o valor referente à farinha de jatobá é inferior; já para a farinha de pequi, o valor é superior. A atividade antioxidante encontrada na farinha de quinoa, de 27,6\% (Tabela 3), foi relativamente menor do que o valor encontrado para suco de uva branco integral de $42 \%$, e para o suco de uva tinto de $95,7 \%$ (VARGAS et al., 2008).

\subsubsection{Biscoitos com farinha de trigo e farinha de quinoa}

Os biscoitos padrão e com adição de farinha de quinoa foram caracterizados quimicamente e os resultados estão expressos na Tabela 4. A adição de farinha de quinoa não alterou o valor energético das formulações dos biscoitos. Em relação à quantidade de carboidratos nas formulações, os biscoitos com 20 e $30 \%$ de farinha de quinoa apresentaram diminuição deste nutriente, conforme se aumentou a concentração da farinha, pois a farinha de quinoa apresenta menor teor deste nutriente.

Observou-se aumento estatisticamente significativo na quantidade de proteína, quando se adicionou 30\% de farinha de quinoa. O acréscimo deste nutriente pode colaborar para a melhora do estado nutricional, pois se sabe que a quinoa fornece aminoácidos importantes para o organismo.

Os resultados encontrados mostram que a adição de farinha de quinoa em biscoitos não causa aumento significativo quanto ao teor de lipídios, quando comparados ao padrão. Um aumento na concentração de lipídios pode não ser interessante em biscoitos, pois pode alterar as características sensoriais, causar perda do valor nutritivo e comercial, em função da oxidação lipídica (BARRERA-ARELLANO, 1993). Em relação aos teores de fibra, pode-se verificar que, apenas na formulação com substituição de $30 \%$ de farinha de quinoa, houve aumento significativo em relação ao padrão Borges et al. (2006) citam o efeito benéfico do consumo de fibras alimentares, sendo que o consumo de aproximadamente $15 \mathrm{~g}$ /dia de fibras solúveis é suficiente para reduzir os níveis sanguíneos de glicose e colesterol em cerca de $10 \%$. Todos os biscoitos apresentaram valores de umidade inferiores a 14\%, ou seja, dentro dos padrões estipulados pela Comissão Nacional de Normas e Padrões de Alimentos (BRASIL, 1978), que é de 14\%. Não houve diferenças significativas entre os biscoitos padrão e aqueles formulados com farinha de quinoa.

Em relação aos teores de polifenóis totais, houve aumento significativo, conforme se acrescentou a farinha de quinoa, até $20 \%$, não havendo diferença quando se adicionaram 30\%. A atividade antioxidante dos biscoitos foi influenciada significativamente apenas quando foram adicionados 30\% de farinha de quinoa.

Tabela 4. Composição centesimal, polifenóis totais e atividade antioxidante dos biscoitos com farinha de trigo e com farinha quinoa.

\begin{tabular}{|c|c|c|c|c|}
\hline & Padrão & $10 \%$ & $20 \%$ & $30 \%$ \\
\hline Valor energético (kcal/100g) & 349 & 350 & 347 & 344 \\
\hline Carboidrato $\left(\mathrm{g} .100 \mathrm{~g}^{-1}\right)$ & 72,9 & 72,1 & 70,9 & 67,6 \\
\hline Proteína $\left(\mathrm{g} .100 \mathrm{~g}^{-1}\right)$ & $9,8 \pm 0,5^{b}$ & $9,9 \pm 0,4^{b}$ & $10,3 \pm 0,1^{b}$ & $12,7 \pm 0,7^{a}$ \\
\hline Lipídios $\left(\mathrm{g} .100 \mathrm{~g}^{-1}\right)$ & $2,03 \pm 0,3^{\text {ns }}$ & $2,44 \pm 0,68$ & $2,4 \pm 0,2$ & $2,5 \pm 1,03$ \\
\hline Fibra bruta $\left(\mathrm{g} .100 \mathrm{~g}^{-1}\right)$ & $0,89 \pm 0,06^{b}$ & $1,32 \pm 0,25^{a b}$ & $1,53 \pm 0,12^{\mathrm{ab}}$ & $1,9 \pm 0,66^{a}$ \\
\hline Cinzas $\left(g .100 g^{-1}\right)$ & $1,34 \pm 0,4^{b}$ & $1,15 \pm 0,09^{b}$ & $1,54 \pm 0,18^{b}$ & $2,19 \pm 0,34^{a}$ \\
\hline Umidade $\left(\mathrm{g} .100 \mathrm{~g}^{-1}\right)$ & $12,96 \pm 0,26^{\text {ns }}$ & $13,06 \pm 0,18$ & $13,23 \pm 0,16$ & $13,13 \pm 0,25$ \\
\hline Polifenóis totais $\left(\mathrm{mg} \cdot 100 \mathrm{~g}^{-1}\right)$ & $39,7 \pm 2,2^{c}$ & $46,2 \pm 2^{b}$ & $58,2 \pm 4,9^{a}$ & $61,3 \pm 2,5^{a}$ \\
\hline Atividade antioxidante (\%SRL) & $7,8 \pm 0,6^{b}$ & $7,6 \pm 0,8^{b}$ & $7,7 \pm 1,6^{b}$ & $9,7 \pm 0,1^{\mathrm{a}}$ \\
\hline
\end{tabular}

Média \pm desvio padrão. Médias seguidas de letras iguais na linha não diferem estatisticamente pelo teste de Duncan $(p<0,05)$. ns $=$ não significativo. $\% \mathrm{SRL}=$ sequestro de radicais livres. 
Biscoitos com diferentes concentrações de farinha de quinoa em substituição parcial à farinha de trigo

BICK, M. A. et al.

Tabela 5. Média e desvio-padrão (DP)* dos quesitos sensoriais dos biscoitos de quinoa.

\begin{tabular}{ccccccc}
\hline Tratamento & Aparência & Cor & Aroma & Textura & Sabor \\
\hline $10 \%$ & $7,14 \pm 1,63^{\text {ns }}$ & $6,96 \pm 1,71^{\text {ns }}$ & $6,88 \pm 1,67^{\text {ns }}$ & $6,52 \pm 1,57^{\text {ns }}$ & $7,18 \pm 1,53^{\text {ns }}$ \\
$20 \%$ & $6,96 \pm 1,87$ & $6,88 \pm 1,80$ & $6,84 \pm 1,48$ & $6,64 \pm 1,70$ & $7,10 \pm 1,64$ \\
$30 \%$ & $7,38 \pm 1,56$ & $7,34 \pm 1,51$ & $7,44 \pm 1,20$ & $7,14 \pm 1,51$ & $7,68 \pm 1,32$ \\
\hline
\end{tabular}

*Escala: 1-Desgostei muitíssimo; 5-Não gostei nem desgostei; 9-Gostei muitíssimo. Média \pm desvio padrão. ns = não significativo pelo teste de Duncan $(p<0,05)$

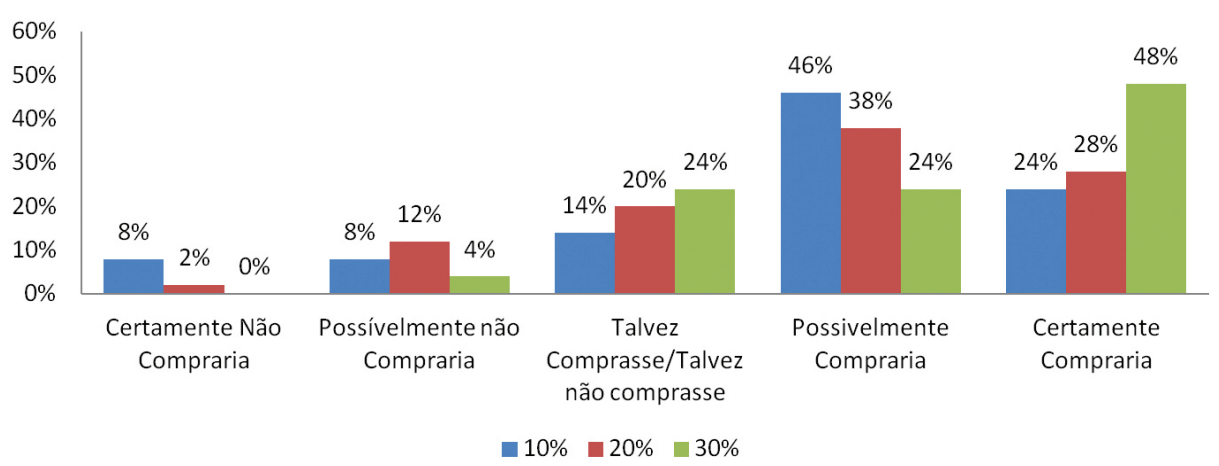

Figura 1. Distribuição de frequência dos provadores para o atributo de intenção de compra dos biscoitos.

Estes resultados demonstram que, mesmo após o processamento, os compostos fenólicos e a atividade antioxidante permanecem no produto, podendo beneficiar os consumidores.

\subsection{Análise sensorial}

Verificou-se na Tabela 5 que os biscoitos foram bem aceitos pelos provadores, apresentando notas superiores a 6 (gostei ligeiramente) para todos os atributos sensoriais.

Não foram observadas diferenças significativas $(p>0,05)$ de aceitabilidade entre os biscoitos com formulações de 10, 20 e 30\% de farinha de quinoa. Portanto, é possível afirmar que a adição de até 30\% de farinha de quinoa em biscoitos não altera suas características sensoriais de aceitabilidade, em relação aos biscoitos com quantidades menores desta farinha. De acordo com Monteiro (1984), para considerar que um produto foi bem aceito, o índice de aceitabilidade deve ser igual ou maior a $70 \%$. Este índice é calculado levando-se em consideração o percentual em relação à nota máxima que o produto poderia receber (nove). Portanto, os três biscoitos apresentaram índice de aceitabilidade superior a $70 \%$ em todos os atributos avaliados, indicando a viabilidade da utilização da farinha de quinoa na produção e comercialização de biscoitos.

Quanto à intenção de compra (Figura 1), 48\% dos provadores certamente comprariam e $24 \%$ dos provadores possivelmente comprariam o biscoito com $30 \%$ de farinha de quinoa, sendo que este tratamento também apresentou menores percentuais na faixa de rejeição. Os biscoitos com adição de 10\% e 20\% de farinha de quinoa apresentaram menores percentuais de intenção de compra positiva (certamente e possivelmente comprariam).

\section{Conclusões}

A farinha de quinoa apresentou boa incorporação à massa dos biscoitos, o que é confirmado pelo fato de os parâmetros físicos observados nos biscoitos experimentais apresentarem valores semelhantes ao biscoito padrão, com exceção da característica 'dureza', que diminuiu à medida que aumentou a concentração da farinha de quinoa, o que possibilitou uma melhora desta característica.

A farinha de quinoa apresentou maiores teores de proteína, lipídios, fibras e minerais, em comparação à farinha de trigo, o que torna interessante a sua utilização, principalmente para grupos que apresentem carências nutricionais.

Observou-se melhora nutricional com a adição de farinha de quinoa nos biscoitos, aumentando os valores de proteínas, lipídios, fibras, cinzas, polifenóis e atividade antioxidante, em comparação ao biscoito padrão. A análise sensorial indicou a possibilidade de comercialização deste produto, tendo em vista o grande percentual de aceitabilidade, em todos os aspectos avaliados, para o produto com adição de até $30 \%$ de farinha de quinoa.

\section{Agradecimentos}

À Capes - Programa Pró-Equipamentos Institucional, Edital $n^{\circ} 13 / 2008$. 
Biscoitos com diferentes concentrações de farinha de quinoa em substituição parcial à farinha de trigo BICK, M. A. et al.

\section{Referências}

AMERICAN ASSOCIATION OF CEREAL CHEMISTS - AACC. Approved methods. 9th. ed. Saint Paul, 1995

AMERICAN ASSOCIATION OF CEREAL CHEMISTS - AACC. Approved methods. 10th. ed. Saint Paul, 2000.

ASCHERI, J. L. R.; NASCIMENTO, R. E.; SPEHAR, C. R. Composição química comparativa de farinha instantânea de quinoa, arroz e milho. Rio de Janeiro: Embrapa, 2002. 4 p. (Comunicado Técnico, 52). Disponível em: <www.infoteca. cnptia.embrapa.br/bitstream/doc/415522/1/ct522002.pdf>.

ASSIS, L. M.; ZAVAREZE, E. R.; RADÜNZ, A. L.; DIAS, Á. R. G.; GUTKOSKI, L. C.; ELIAS, M. C. Propriedades nutricionais, tecnológicas e sensoriais de biscoitos com substituição de farinha de trigo por farinha de aveia ou farinha de arroz parboilizado. Alimentos e Nutrição, Araraquara, v. 20, n. 1, p. 15-24, 2009.

CUNNIFF, P. A. (Ed.). Official Methods of Analysis of the Association of Official Analytical Chemists. 16th ed. Washington: AOAC, 1995. (v. 2).

BARRERA-ARELLANO, D. Estabilidade de óleos e gorduras. Revista Óleos e Grãos, São Bernardo do Campo, n. 13, p. 10-13, 1993.

BORGES, J. T. S.; BORGES, J. T. S.; ASCHERI, J. L. R.; ASCHERI, D. R.; NASCIMENTO, R. E.; FREITAS, A. S. Propriedades de cozimento e caracterização físico-química de macarrão pré-cozido a base de farinha integral de quinoa (Chenopodium quinoa, Willd) e farinha de arroz (Oryza sativa, L) polido por extrusão termoplástica. Boletim CEPPA, Curitiba, v. 21, n. 2, p. 303-322, 2003

BORGES, J. T. S.; PIROZI, M. R.; DELLA LUCIA, S. M.; PEREIRA, P. C.; MORAES, A. R. F.; CASTRO, V. C. Utilização de farinha mista de aveia e trigo na elaboração de bolos. Boletim CEPPA, Curitiba, v. 24, n. 1, p. 145-162, 2006

BRADY, K. HO, C.; ROSEN, R. T.; SANG, S.; KARWE, M. V. Effects of processing on the nutraceutical profile of quinoa. Food Chemistry, London, v. 100, n. 3, p. 1209-1216, 2007

BRASIL. Portaria n ${ }^{\circ}$ 108, de 4 de setembro de 1991. Normas gerais de amostragem para análise de rotina. Método número 11 - Fibra Bruta. Diário Oficial [da] República do Brasil, Poder Executivo, Brasília, DF, 17 set. de 1991. Seção 1, p. 19813.

BRASIL. Resolução n 12, de 24 julho de 1978. A CNNPA do Ministério da Saúde aprova 47 padrões de identidade e qualidade relativos a alimentos e bebidas para serem seguidos em todo território brasileiro. Diário Oficial [da] República do Brasil, Poder Executivo, Brasília, DF, 1978. Seção 1.

BRASIL. Resolução RDC, n 263 de 22 de setembro de 2005. Aprova o Regulamento Técnico para produtos de cereais, amidos, farinhas e farelos, constantes do anexo desta Portaria.
Diário Oficial [da] República do Brasil, Poder Executivo, Brasília, DF, 23 set. 2005.

CALDERELLI, V. A. S.; BENASSI, M. T.; VISENTAINER, J. V.; MATIOLI, G. Quinoa and flaxseed: potential ingredients in the production of bread with functional quality. Brazilian Archives of Biology and Technology, Curitiba, v. 53, n. 4, p. 981-986, 2010.

CAPRILES, V. D.; COELHO, K. D.; MATIAS, A. C. G.; ARÊAS, J. A. G. Efeito da adição de amaranto na composição e na aceitabilidade de biscoitos tipo cookie e do pão de forma. Alimentos de Nutrição, Araraquara, v. 17, n. 3, p. 269-274, 2006.

COSTA, A. M.; ROQUE-SPECHT, V. F.; CELESTINO, S. M. C.; CABRAL, Y. O. E.; CABRAL, D. O. E.; DINIZ, J. D. A. S. Estabilidade de compostos fenólicos e flavonóides nas farinhas de jatobá e pequi processadas em diferentes temperaturas. In: CONGRESSO BRASILEIRO DE FRUTICULTURA, 22., 2012, Bento Gonçalves. Anais... Bento Gonçalves: SBF, 2012.

DUTCOSKY, S. D. Análise sensorial de alimentos. 3. ed. Curitiba: Champagnat, 2011.

FARINAZZI-MACHADO, F. M. V.; BARBALHO, S. M.; OSHIIWA, M.; GOULART, R.; PESSAN JUNIOR, O. Use of cereal bars with quinoa (Chenopodium quinoa $\mathrm{W}$.) to reduce risk factors related to cardiovascular diseases. Ciência e Tecnologia dos Alimentos, Campinas, v. 32, n. 2, p. 239-244, 2012.

FASOLIN, L. H.; ALMEIDA, G. C.; CASTANHO, P. S.; NETTOOLIVEIRA, E. R. Biscoitos produzidos com farinha de banana: avaliações químicas, físicas e sensorial. Ciência e Tecnologia dos Alimentos, Campinas, v. 27, n. 3, p. 524-529, 2007.

FERREIRA, S. M. R.; LUPARELLI, P. C.; SCHIEFERDECKER, M. E. M.; VILELA, R. M. Cookies sem glúten a partir da farinha de sorgo. Archivos Latinoamericanos de Nutricion, Caracas, v. 59, n. 4, p. 433-440, 2009.

HIROSE, Y.; FUJITA, T.; ISHII, T.; UENO, N. Antioxidative properties and flavonoid composition of Chenopodium quinoa seeds cultivated in Japan. Food Chemistry, London, v. 119, n. 4, p. 1330-1306, 2010.

JAMES, L. E. A. Quinoa (Chenopodium quinoa Willd.): composition, chemistry, nutritional and functional properties. Advances in Food and Nutrition Research, Chile, v. 58, n. 9, p. 1043-4526, 2009.

KUSKOSKI, E. M.; ASUERO, A. G.; MORALES, M. T.; FETT, R. Frutos tropicais silvestres e polpas congeladas: atividade antioxidante, polifenóis e antocianinas. Ciência Rural, Santa Maria, v. 36, n. 4, p. 1283-1287, 2006.

LOPES, C. O. Aproveitamento, composição nutricional e antinutricional da farinha de quinoa. Alimentos e Nutrição, Araraquara, v. 20, n. 4, p. 669-675, 2009. 
Biscoitos com diferentes concentrações de farinha de quinoa em substituição parcial à farinha de trigo BICK, M. A. et al.

MENDEZ, M. H. M.; FERNANDES, M. L.; RODRIGUES, M. C. R.; DERIVI, S. C. N. Tabela de Composição de Alimentos. Niterói: Editora da Universidade Federal Fluminense, 1995. p. 41.

MONTEIRO, C. L. B. Técnicas de avaliação sensorial. 2. ed. Curitiba: CEPPA, 1984. $101 \mathrm{p}$.

MORAES, K. S.; ZAVAREZE, E. R.; MIRANDA, M. Z.; SALASMELLADO, M. M. Avaliação tecnológica de biscoitos tipo cookie com variações nos teores de lipídios e de açúcar. Ciência e Tecnologia de Alimentos, Campinas, v. 30, p. 233-242, 2010.

ORGANIZAÇÃO MUNDIAL DA SAÚDE - OMS. Manual das necessidades nutricionais humanas. São Paulo: Atheneu, 2003.

REPO-CARRASCO-VALENCIA, R. A. M.; HELLSTRÖM, J. K.; PIHLAVA, J.; MATTILA, P. H. Flavonoids and other phenolic compounds in Andean indigenous grains: Quinoa (Chenopodium quinoa), kaniwa (Chenopodium pallidicaule) and kiwicha (Amaranthus caudatus). Food Chemistry, London, v. 120, n. 1, p. 128-133, 2010.

REPO-CARRASCO-VALENCIA, R. A. M.; SERNA, L. A. Quinoa (Chenopodium quinoa Willd.) as a source of dietery fiber and other functional components. Ciência e Tecnologia de Alimentos, Campinas, v. 31, n. 1, p. 225-230, 2011.

RUFINO, M. S. M.; ALVES, R. E.; BRITO, E. S.; MORAIS, S. M.; SAMPAIO, C. G.; PÉREZ-JIMÉNEZ, J.; SAURA-CALIXTO, F. $D$. Determinação da atividade antioxidante total em frutas pela captura do radical livre ABTS. Fortaleza: Embrapa Agroindústria Tropical, 2007. (Comunicado Técnico, 128).

STIKIC, R.; DJORDJE, G.; MIRJANA, D.; VUCELIC-RADOVIC, B.; JOVANOVIC, Z.; MILOJKOVIC-OPSENICA, D.; JACOBSEN, S.; MILOVANOVIC, M. Agronomic and nutritional evaluation of quinoa seeds (Chenopodium quinoa Wild.) as an ingredient in bread formulations. Journal of Cereal Science, London, v. 55, n. 2, p. 132-138, 2012.

SILVA, F. D.; PANTE, C. F.; PRUDÊNCIO, S. H.; RIBEIRO, A. B. Elaboração de uma barra de cereal de quinoa e suas propriedades sensoriais e nutricionais. Alimentos e Nutrição, Araraquara, v. 22, n. 1, p. 63-69, 2011.

SINGLETON, V. L.; ROSSI JUNIOR, J. A. Colorimetry of total phenolics with phosphomolybdic-phosphotungstic acid reagents. American Journal of Enology and Viticulture, Davis, v. 16, p. 144-158, 1965.

SOUZA, V. R.; PEREIRA, P. A. P.; SILVA, T. L. T. .; LIMA, L. C. O.; $\mathrm{PIO}, \mathrm{R}$. Determination of the bioactive compounds, antioxidant activity and chemical composition of Brazilian blackberry, red raspberry, strawberry, blueberry and sweet cherry fruits. Food Chemistry, London, v. 156, p. 362-368, 2014.

UNIVERSIDADE ESTADUAL DE CAMPINAS - UNICAMP. Tabela Brasileira de Composição de Alimentos - TACO. 4. ed. Campinas: UNICAMP/NEPA, 2011. $161 \mathrm{p}$.

VARGAS, P. N.; HOELZEL, S. C.; ROSA, C. S. Determinação do teor de polifenóis totais e atividade antioxidante em sucos de uva comerciais. Alimentos e Nutrição, Araraquara, v. 19, n. 1 , p. 11-15, 2008.

VILLARROEL, M.; HUIRIQUEO, C.; HAZBUN, J.; CARRILLO, D. Desarrollo de una formulación optimizada de galletas para celiacos utilizando harina desgrasada de avellana chilena (Gevuina avellana, Mol) y harina de quinoa (Chenopodium quinoa Willd). Archivos Latinoamericanos de Nutrición, Caracas, v. 59, n. 2, p. 184-190, 2009.

ZENEBON, O.; PASCUET, N. S. (Coord.). Métodos físicoquímicos para análise de alimentos. 4. ed. Brasília: Ministério da Saúde; São Paulo: Instituto Adolfo Lutz, 2005. 1018 p. 